\title{
Time resolved nanosecond imaging of the propagation of a corona-like plasma discharge in water at positive applied voltage polarity
}

\author{
P.H. Ceccato ${ }^{\xi 1}$, O. Guaitella ${ }^{1}$, M. Rabec Le Gloahec ${ }^{2}$, A. Rousseau ${ }^{1}$ \\ ${ }^{1}$ LPP Ecole Polytechnique CNRS Palaiseau, France \\ ${ }^{2}$ LULI Ecole Polytechnique CNRS Palaiseau, France
}

E-mail: paul.ceccato@1pp.polytechnique.fr

\begin{abstract}
The present paper is an experimental study of a pulsed filamentary plasma discharge inside liquid water in pin to plane electrode configuration. Time resolved electrical and imaging diagnostics have been performed. The initiation and the propagation of the discharge have been studied for several experimental parameters. The propagation is continuous and is followed by reilluminations at low water conductivity. The measured propagation velocity of the plasma discharge is $30 \mathrm{~km} / \mathrm{s}$ for the secondary positive mode. This velocity was found to be surprisingly constant whatever the experimental parameters and especially as a function of the water conductivity.
\end{abstract}

Keywords. dielectric liquids, water, high voltage insulation, pulsed discharge, prebreakdown phenomena, plasma, filamentary discharge, streamer, streak imaging, fast nanosecond imaging, intensified CCD camera, time resolved, initiation, inception, propagation velocity, time lag, breakdown.

Subject classification numbers. 52.80.Wq, 77.22.Jp, 72.20.Ht

Submitted to. Journal of Physics D: Applied Physics

\section{Introduction}

Plasma discharges in water have been studied for decades in pulsed power applications [1-3] [5-9]. Even with a quite high conductivity the breakdown strength of water and its exceptional dielectric constant have been noticed early and applied for water insulated high voltage lines. The high dielectric permittivity allows to construct compact high voltage transmission lines and the ability of liquid water to withstand high voltages during hundreds of nanoseconds is used for high voltage insulation or high voltage switching. In such systems, breakdown occurs when plasma filaments propagate across the dielectric liquid and bridge the interelectrode gap.

More recently both thermal and non thermal plasma discharges inside water have been proved efficient for pollution removal and sterilization in environmental [10-17] or medical applications [1819]. The plasma discharge is able to break water molecule and produce highly chemically reactive radicals such as $\mathrm{H}, \mathrm{O}$ and $\mathrm{OH}$ (and then $\mathrm{H}_{2} \mathrm{O}_{2}$ ) both in the ionized gas channels and in the liquid around the plasma filaments. Those radicals are able to oxidize any organic molecule dissolved into the liquid into $\mathrm{H}_{2} \mathrm{O} C \mathrm{C}$ and $\mathrm{CO}_{2}$ with a minimum of byproducts and maximum energy efficiency. The main problem from the chemical application point of view is to minimize the byproducts and maximize the energy efficiency. This requires to understand the primary source of radicals produced by the plasma and to characterize the subsequent chemical reactions initiated by this source of radicals. The production of the active species directly depends on the plasma parameters, namely the electron density and temperature, the gas density and temperature but also on the discharge morphology and duration. Consequently, a more detailed understanding of the physics of plasma discharges in dense medium (condensed matter) is required. In the present paper, fast electrical and nanosecond imaging diagnostics have been implemented and a parametric study has been performed in order to characterize the discharge from the physical point of view.

\footnotetext{
گ email: paul.ceccato@lpp.polytechnique.fr
} 
The main problem with discharges inside water (and in a general way for discharges inside liquids) is whether or not there is an electron avalanche possible in the liquid phase or if a phase change is required for the avalanches to occur. The plasma state inside a liquid is either a peculiar state of locally conductive condensed matter in electron avalanche regime (as in avalanche semiconductors), or on the contrary an ionized gas phase resulting from vaporization and ionization of the dense medium. The liquid phase main difference with gases is the density scaling which leads very high collision frequency and energy dissipation rate and thus to a low electronic mobility. Accordingly some numerical fluid simulations has been performed by some authors [20-23] with such a "dense gas" approximation (or extrapolation). They concluded that, a liquid phase will require tremendous electric field in the hundreds of $\mathrm{MV} / \mathrm{cm}$ to ionize, accelerate and multiply an initial seed electron into an electronic avalanche. Thus there need to be phase change [17] or density lowering [20] or microbubble impurities breakdown [17] [21] or electrostatic crack mechanism [25-27] prior to electron avalanches. One need to be aware that such fluid simulations [20-23] do not take into account any phase change or charge exchange trough a plasma/liquid interface or the fact that charges carriers (and thus the charge mobility) are not necessarily the same inside the plasma and inside the liquid.

Some authors have investigated plasma discharges inside noble gas liquids [28-30] where the electron mobility is much higher than in organic liquids [31-35] [44]. Noble gas liquids are usually diatomic which is convenient for spectroscopic studies. Studies of plasma discharge inside organic liquid focus on the influence of thermodynamic or fluid parameters such as viscosity [36-43]. A particularly interesting parameter is the addition of high electron affinity additives, or low ionization potential additives. It has been found that a large electron affinity additive favors the discharges under negative applied voltage polarity whereas low ionization level additives (such as $\mathrm{CCl}_{4}$ in oil) favor the positive polarity discharge [48-50]. The negative discharge is often considered to be the result of electrostatic and fluid motion processes [36-43]. The positive discharge due to its very high velocity and its filamentary shape is likely to follow a streamer-like [51-52] mechanism with field ionization of water molecule at the filament tip [2] [53].

The energy deposition leading to the formation of the plasma cavity and the subsequent interaction of the plasma and the cavity interface leads to the specific behaviour of plasmas inside liquids compared to gas-phase plasmas. Several modes of plasma have been observed in literature and at least four modes have been reported for positive polarity in typical transformer oil: [35] [48]. Each mode leads (in water) to very different plasma parameters (ne, Te, Tgas, Pgas) and a different chemical yield [1011] [15-16] [54]. Those several modes can be obtained by changing the experimental parameters such as the electrode configuration and the applied voltage, or by gas injection nearby the electrodes. The initiation and the propagation mechanism of each mode are still not well known. Several theories have been proposed in the literature but a global understanding has not been achieved yet.

The present study focuses on the case of water and investigates in particular the influence of water conductivity. The purpose of this paper is to give an experimental insight about those initiation and propagation mechanisms of the plasma filaments through the water at positive applied voltage polarity.

The structure of the present paper is as follows. In section 2 are presented the pin to plane reactor, the electrical power supply and diagnostics, and the iCCD and streak diagnostics. In section 3 is presented a description of the time resolved growth of the discharge in relation to the discharge current. In section 4 is presented the initiation phase of the discharge between the beginning of the applied voltage pulse and the beginning of the propagation of the plasma filaments and the several stages of the discharge propagation. In section is 5 is discussed the influence of the water conductivity on the propagation velocity of the discharge across the interelectrode gap.

\section{Experimental setup}

A pin to plane electrode configuration figure 1 has been used. The tungsten pin has $50 \mu \mathrm{m}$ curvature radius in order to obtain sufficient electric field amplification near the pin electrode for discharge initiation at the maximum applied voltage of $40 \mathrm{kV}$ of our power supply. The interelectrode gap is typically of the order of $1 \mathrm{~cm}$ in most of the experimental results presented in this paper and can be changed from $2 \mathrm{~mm}$ to $5 \mathrm{~cm}$. Thus the present discharge configuration can be qualified as long gap and inhomogeneous electric field in opposition to plane to plane homogeneous with submilimeter gaps classically found in switches studies [1-3] [6-8]. The HV pin electrode is insulated with epoxy 
dielectric except for the last $2 \mathrm{~mm}$ to avoid resistive losses. Special care should be taken to avoid discharge initiation at the triple point instead of the pin electrode. The Teflon reactor figure 1 is filled with $1 \mathrm{~L}$ of water and the ionic conductivity of the water is adjusted in the $7 \mu \mathrm{S} / \mathrm{cm}-1 \mathrm{mS} / \mathrm{cm}$ range with phosphate salt or sodium salt.

The power supply is a homemade Marx generator with adjustable storage capacitor bank composed of $2 \mathrm{nF}$ ceramic capacitors. The switching element is a pressurized self-triggered spark gap. The rise time is $20 \mathrm{~ns}$, with peak amplitude of typically $40 \mathrm{kV}$ and a fall time depending on the reactor resistivity. The voltage level is adjusted by changing the gas pressure in the spark gap. The storage capacitor of the power supply has been changed to adjust the voltage pulse duration figure 9a. The pulse has a typical RC decay shape. Pulse duration (or halfwidth) is critical to control the duration of the plasma discharge and avoid sparking figure 9 a figure 7.

A Lecroy PPE20kV capacitive divider probe was used to measure the voltage waveform. A Pearson $65851 \mathrm{GHz}$ wide band current transformer was used to measure the discharge current on the return path from the plane electrode to the ground. Since the power supply is self-triggered, the discharge current measure is used for triggering the optical diagnostics.

The imaging system consists of two Andor iCCDs IstarDH734 observing the light emission of the plasma discharge trough a beam splitter figure $2 b$. Such dual iCCD system figure 2 a figure $2 b$ allows to obtain two successive images on the same applied voltage pulse figure $2 \mathrm{c}$ and thus allow us to bypass the single gate per iCCD limitation. With this setup, a fine time resolved study can be performed even if the discharge pattern is not reproducible from one shot to another figure 5. The intrinsic delay of the triggering apparatus is 220ns. The delay of the iCCDs is adjusted from the beginning of the voltage pulse at $\mathrm{t}=0$ in figure $2 \mathrm{c}$, figure $4 \mathrm{a}$, figure $4 \mathrm{~b}$, and figure $4 \mathrm{c}$. The delay difference between the two iCCDs is typically of the order of a few nanoseconds or a few tens of nanoseconds depending on the propagation velocity of the phenomena to observe: $2 \mathrm{~ns}-10 \mathrm{~ns}$ difference can be used to see fast events, 30ns difference is suitable for the discharge propagation, and longer gate spacing of typically $2 \mu \mathrm{s}$ can be used for the gas motion (not presented here). Measurements have been made on single shot images with two successive gates per applied voltage pulse in, figure 10, and figure 12 .

A streak camera was used figure 3 to confirm the two iCCD setup results. This type of camera allows to observe 1D object during a time sweep. The vertical axis of the output streak image is time and depends on the time sweep range. The time range was adjusted typically to $1 \mu$ s or $10 \mu$ s sweep to observe respectively the propagation figure 8 and the reilluminations figure 13. Horizontal axis of the output streak image is space and corresponds to the axis of the entrance slit. The entrance slit of the streak camera was put along the interelectrode axis as shown in figure 3. The image of the discharge is made on the entrance slit of the streak with an achromatic doublet lens and a magnification factor of one or two. In addition, an iCCD was put on the opposite side of the reactor to check on 2D emission images if one plasma filament is correctly positioned along the streak entrance slit. A careful selection of the images was performed to be sure to observe an event where the filament is propagating along the axis of the entrance slit on the streak camera. The intrinsic delay of the streak camera is typically two times the time range sweep, thus only microsecond sweep range and below was possible in our self-triggered discharge setup.

With emission imaging, the initiation and the propagation of the discharge can be studied. However the non luminous phenomena such as nucleation of bubbles, and the post-discharge gas dynamics are not accessible.

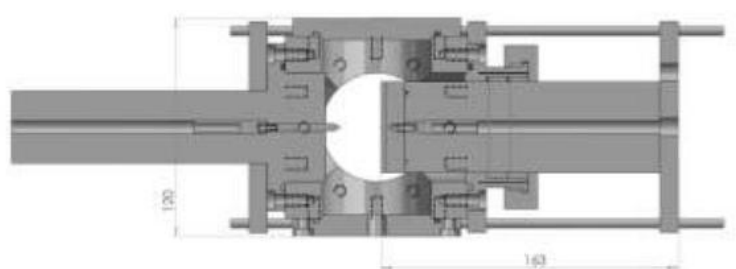

Figure 1. Experimental setup of a pin to plane reactor with a $200 \mu \mathrm{m}$ curvature radius $\mathrm{HV}$ pin and $1 \mathrm{~cm}$ gap, both electrodes are underwater, the plane is coated with a dielectric to avoid breakdown. 


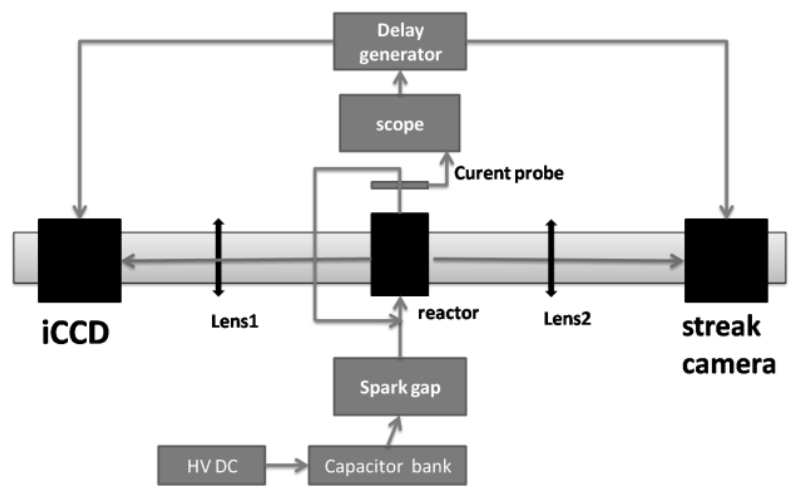

Figure 2a. Time resolved imaging experimental setup with one intensified CCD and a streak camera on each sides of the reactor, the entrance slit of the streak is put along the interelectrode axis as shown in figure 3, for setup with two iCCD the streak is replaced by a second iCCD to obtain two images per plasma shot as shown in figure $2 \mathrm{c}$

iCCD1 gate

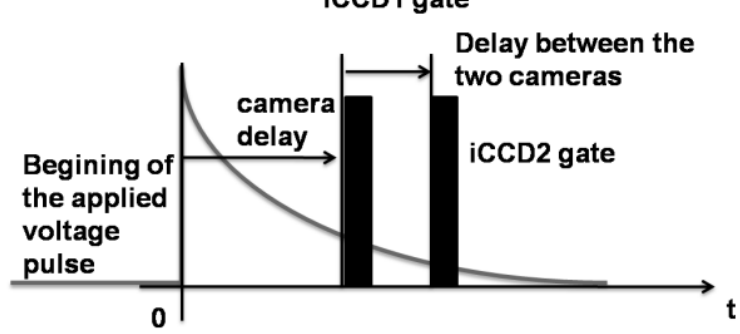

Figure 2c. Delay generator setup to perform time resolved study with two iCCDs, two gates per applied voltage pulse.

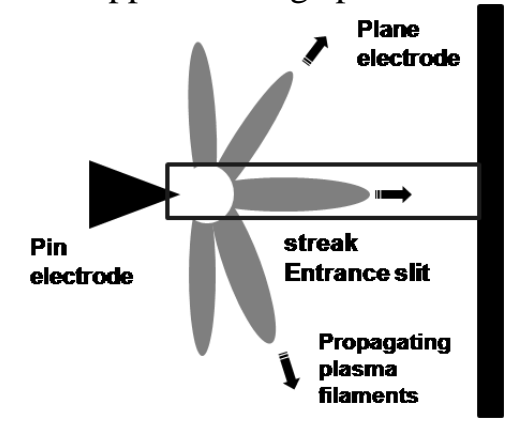

Figure 3. For streak imaging the entrance slit of the streak is along the pin to plane interelectrode axis, emission of the plasma is observed and thus the streak allows to observe the propagation of the plasma

filament during a time sweep over one applied voltage pulse.

3. General description of the time resolved discharge growth in water at positive applied voltage The initiation of the plasma discharge inside water requires some time, typically hundreds of nanoseconds as shown in figure $4 \mathrm{a}$, figure, $4 \mathrm{~b}$ and figure $4 \mathrm{c}$, and can be as much as a few microseconds in our conditions as shown in stage (1) in figure 4a. Here we call "initiation" the time between the beginning of the applied voltage pulse at $\mathrm{t}=0$ stage (0) in figure $4 \mathrm{a}$ and the first light emission from the discharge. The timescale of the initiation can even be longer in figure 4a than the timescale of the propagation itself. There is no plasma yet during this stage. From the initiation current level, electrolysis can be neglected and the injected energy during this initiation phase is consistent with the nucleation of a gas bubble. A more detailed analysis of the initiation delay as a function of voltage and water conductivity in terms of statistics and identification of the non luminous events associated with this initiation delay will be presented in a following paper.

The plasma discharge begins with the propagation of a "primary mode" followed by a "secondary mode" in , this denomination is made according literature [55]. The primary mode is difficult to resolve and is associated to a weak emission with a lot of tiny filaments close to the HV pin electrode. This primary mode always propagates less that a millimeter in our conditions and is not necessarily present at each shot. The beginning of the primary mode is associated to a weak current ramp as can 
be seen on stage (2) in figure 4a that is often masked by the stray capacitive current and its ringing at the beginning of the current waveform stage (0) in figure 4b. This "stray" current superimposed with the initiation current is LC ringing obtained when applying voltage, with L from the power supply and the reactor circuit, and $\mathrm{C}$ from the inter-electrode capacitance.

A sharp current rise of some amperes can be observed at the beginning of the propagation of the secondary mode stage (3) in figure 4afigure $4 \mathrm{~b}$ and an even bigger increase of some kA can be observed when the plasma filaments bridge the interelectrode gap and sparking occurs as shown in figure 7.

During the secondary mode propagation two successive phases can be distinguished at low conductivity water: "continuous phase" as shown in stage (3) of figure $4 \mathrm{a}$, figure $4 \mathrm{~b}$, figure 8 , and "reilluminations" in stage (4) in figure $4 \mathrm{a}$, figure $4 \mathrm{~b}$ and figure 13 . Reilluminations in figure 6 , figure 12 , and figure 13 are associated to current spikes in figure $4 \mathrm{~b}$. Breakdown of the liquid gap is associated with this sparking. Initiation, propagation of the primary mode, and propagation of the secondary mode are usually referred as prebreakdown phenomena. In the present paper, we are investigating this prebreakdown plasma discharge inside the liquid and not the arc discharge phase at breakdown in figure 7 .

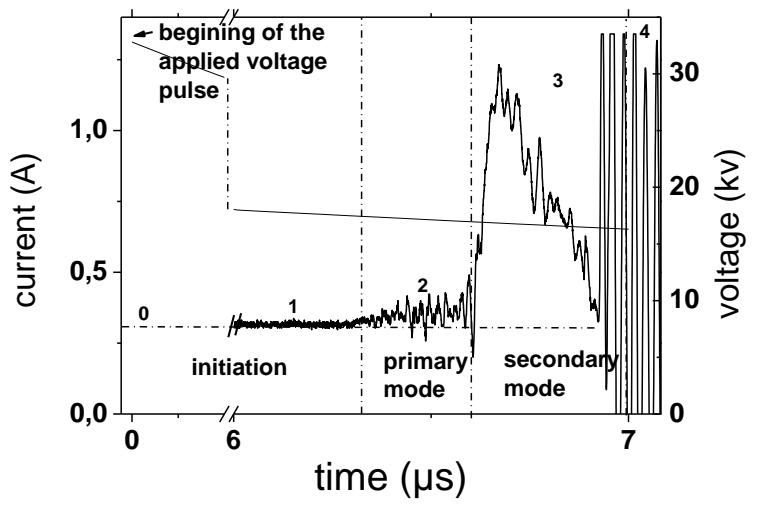

Figure 4a. Zoom on the current waveform, (0) beginning of the applied voltage pulse, (1) initiation (or pre-initiation) current due to conductivity of water $\sim 300 \mathrm{~mA}$ here, (2) current ramp of the plasma primary positive mode $\sim 50 \mathrm{~mA}$, (3) current increase of the secondary positive mode $\sim 1 \mathrm{Amp},(4)$ reilluminations, $(7 \mu \mathrm{S} / \mathrm{cm}, 40 \mathrm{kV})$.

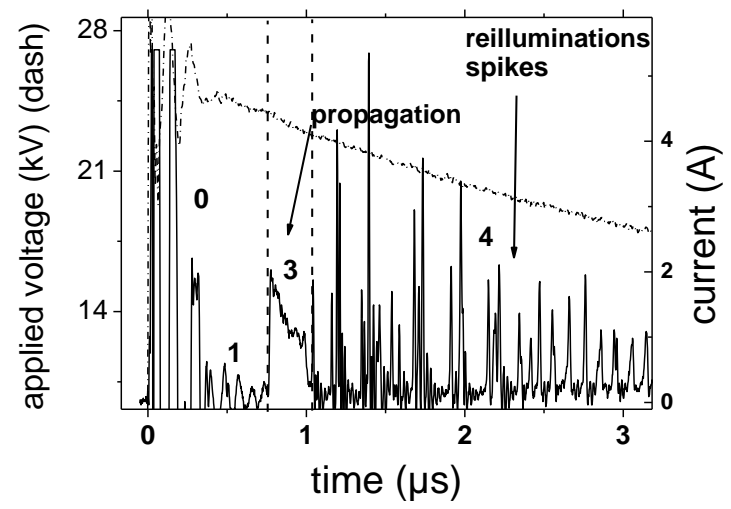

Figure 4b. Current waveform in distilled water, (0) stray displacement current, (1) pre-initiation current due to water conductivity, (3) large pulse of the secondary positive mode followed by (4) reillumination spikes with decaying amplitude, (4cm gap, $40 \mathrm{kV})$. 


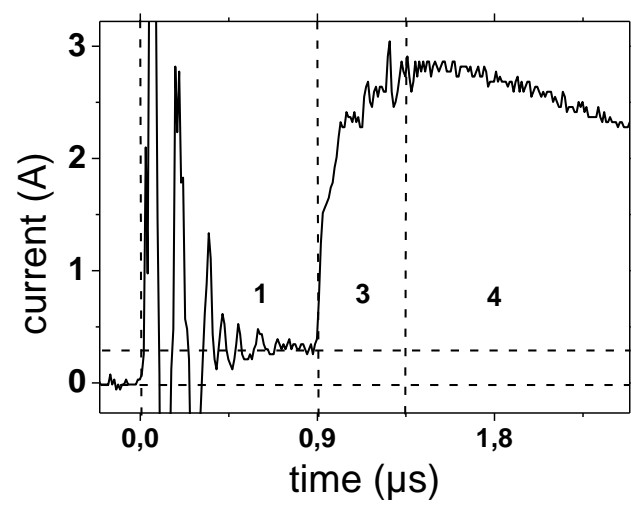

Figure 4c. Current waveform in $500 \mu \mathrm{S} / \mathrm{cm}$ water, there is no reillumination spikes, (1) initiation current, (3) propagation, (4) decay, there is a large current even when the discharge has stopped to propagate and just decay according to the voltage pulse decay, $(4 \mathrm{~cm}$ gap, $40 \mathrm{kV})$.
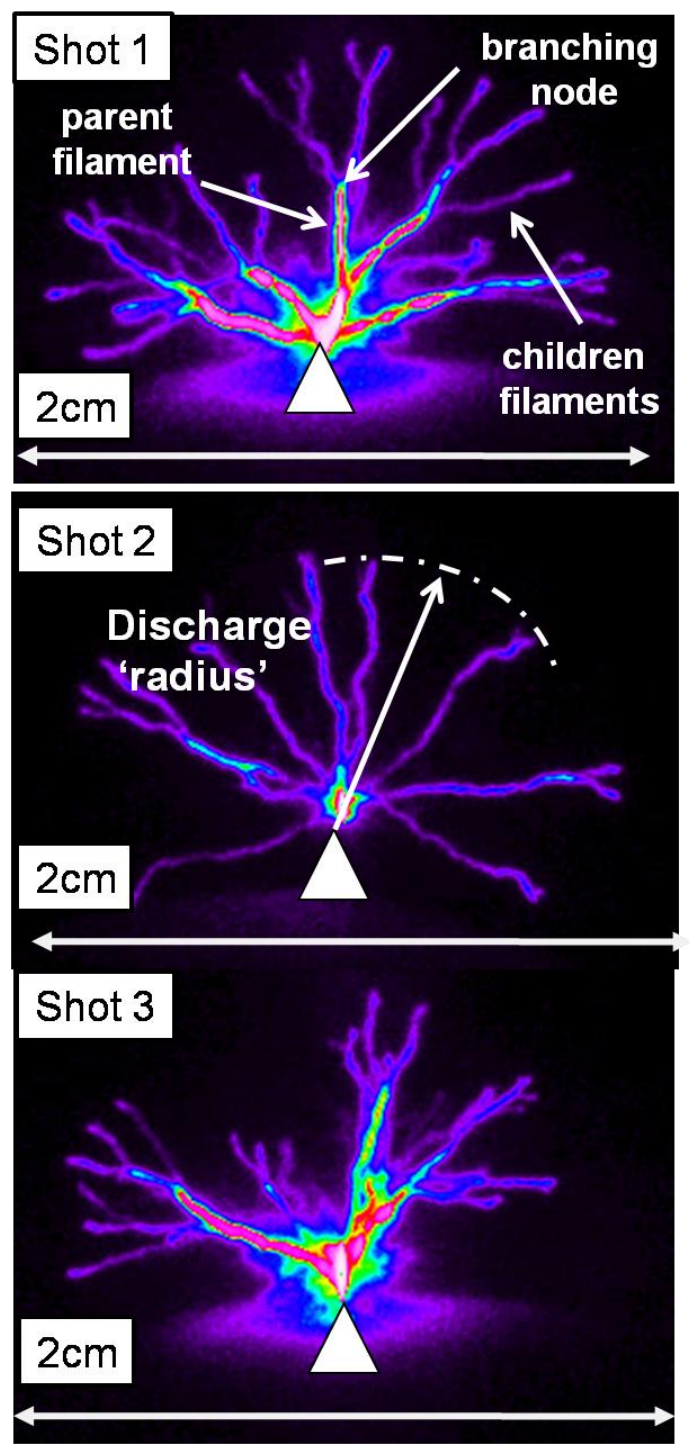

Figure 5. Simultaneous propagation of the plasma filaments on a single shot image, a radius of the discharge can be defined. with $2 \mathrm{~ns}$ gate and $200 \mathrm{~ns}$ delay from $\mathrm{t}=0$, the length at some delay is quite reproducible and allow to use velocity-growth plots. Typical branching patterns observed on several shots in the same experimental conditions, this shows the chaotic behaviour of branching, $(100 \mu \mathrm{S} / \mathrm{cm}$, $40 \mathrm{kV}, 4 \mathrm{~cm}$ gap). 


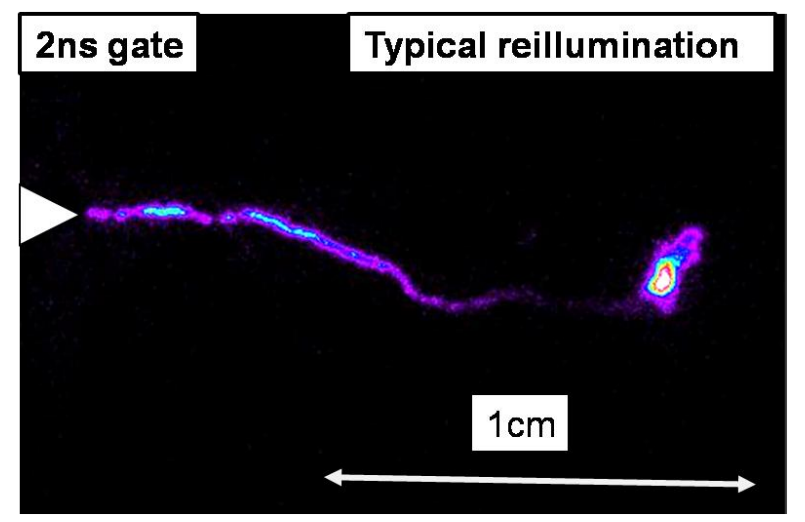

Figure 6. Reillumination in distilled water, the plasma re-uses only one of the gas channel from , the emission intensity of the channel is weak and the tip emission is intense, the tip can be just a spot or a segment, reilluminations are associated to phase (4) in figure $4 \mathrm{a}$ and figure $4 \mathrm{~b},(3 \mu \mathrm{s}$ delay, $2 \mathrm{~ns}$ gate, distilled water, $4 \mathrm{~cm}$ gap, $40 \mathrm{kV}$ ).

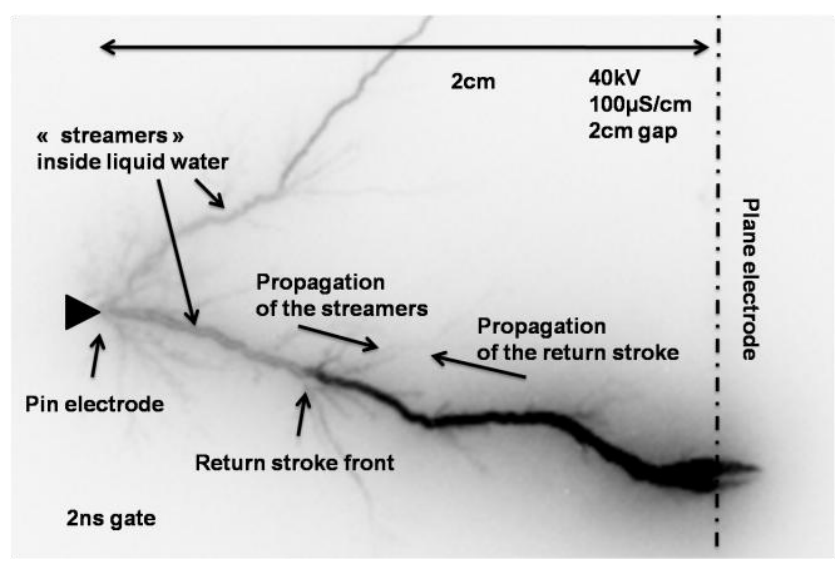

Figure 7. Transition to spark, when the plasma filament reach the opposite plane electrode a thermalization return stoke propagates back to the pin electrode, ( $2 \mathrm{n}$ gate, $4 \mu \mathrm{s}$ delay, distilled water, $40 \mathrm{kV})$.

\section{Detailed characterization of the initiation and the propagation of the discharge}

\subsection{Propagation of the secondary mode and determination of the propagation velocity}

At the transition between the primary to the secondary mode, a bright emission close to the HV pin electrode is observed and the bright filaments of the secondary mode structure propagate in a radial direction from this high intensity spot.

Here we do not rely on time delay to breakdown divided by the interelectrode gap to obtain a mean propagation velocity. This method would be approximate and would not take into account the initiation delay. We resolve the propagation velocity across the gap.

The propagation of the plasma filaments is simultaneous and no velocity difference can be observed between them in. All the filaments start at the same time and propagate at an equal velocity in a radial direction. The envelope of the discharge filaments at this stage is hemispherical and a "radius" or a "length" of the discharge can thus be defined as shown in . With a single iCCD and accumulated images, length vs time plots figure $9 \mathrm{a}$ figure $9 \mathrm{~b}$ can be made by measuring the mean discharge growth as a function of the iCCD gate delay. We observe a linear growth (1) in figure 9a followed by a stopping of the propagation (2) in figure 9a. The discharge velocity can be extracted by derivating this curve.

This method implies that the initiation delay has a reduced jitter and that the propagation duration is sufficiently long compared to the initiation duration. This is only true at high voltage and long pulse duration. For this set of measurements, we used $40 \mathrm{kV}$ applied voltage with $5 \mu$ s halfwidth and interelectrode gap of $4 \mathrm{~cm}$. If those conditions are not met, the figure $9 \mathrm{a}$ and figure $9 \mathrm{~b}$ plot would be corrupted by the jitter statistics. 
The streak imaging indicates a constant and continuous propagation as can be seen by the typical triangular pattern in figure 8 and figure 13. The propagation velocity is obtained by the slope of this triangular pattern. The resolution of those streak images was dictated by the entrance slit aperture and was respectively of $5 \mathrm{~ns}$ and $20 \mathrm{~ns}$ resolution in figure 8 . This study thus demonstrates a continuous propagation on nanosecond scale of the secondary mode.

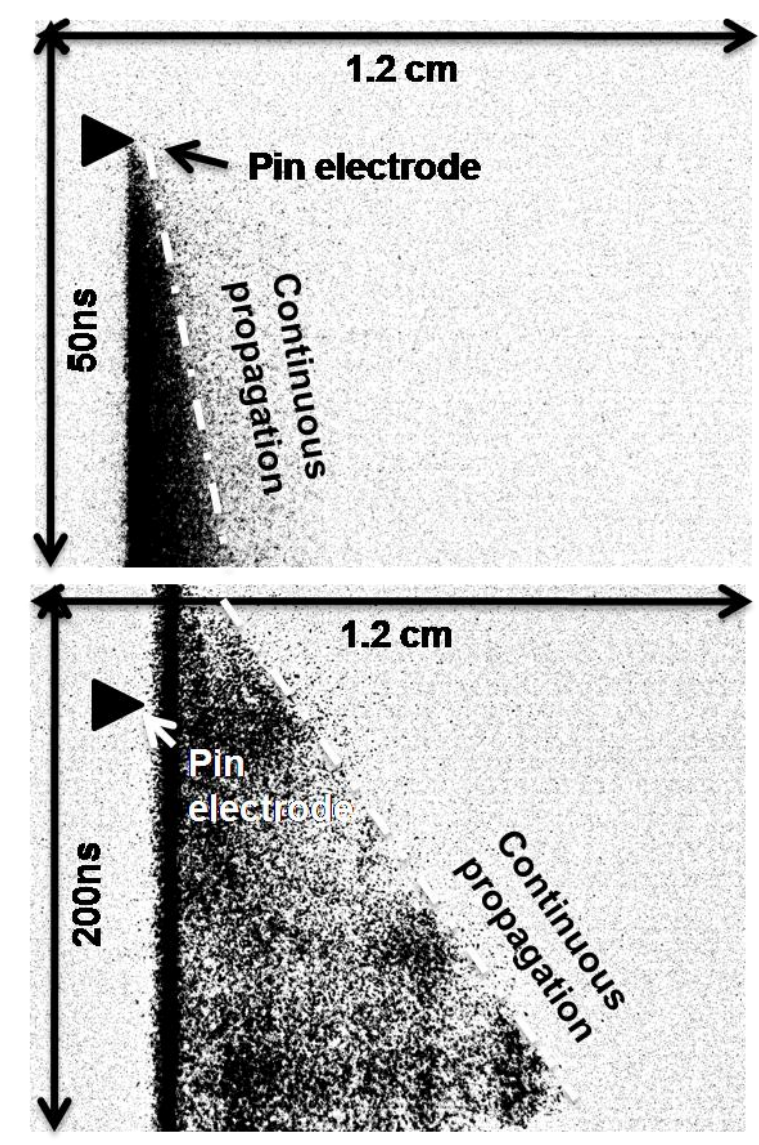

Figure 8. Streak image of the secondary mode propagation, the triangular shape clearly demonstrate constant propagation. $1 \mu \mathrm{s}$ time sweep (vertical axis), $1 \mathrm{~cm}$ horizontal axis, the pin electrode is on the left side ( $40 \mathrm{KV}$, distilled water, $4 \mathrm{~cm}$ gap).

\subsection{Influence of applied voltage and water conductivity on the discharge propagation velocity}

First, we observed that the propagation velocity of the filaments is weakly affected by the voltage level at the time of initiation or the applied voltage decay slope. The voltage level at $t=0$ was changed in a range of $30 \%$ by changing the spark gap pressure and by changing the storage capacitor value in order to verify that the discharge is not sensitive to this RC decay slope.

The main result of this paper is that the propagation velocity has been found to be independent of the liquid ionic conductivity figure $9 \mathrm{~b}$. The stage (1) in figure $9 \mathrm{a}$ and figure $9 \mathrm{~b}$ and also indicate a constant propagation velocity across the gap. 


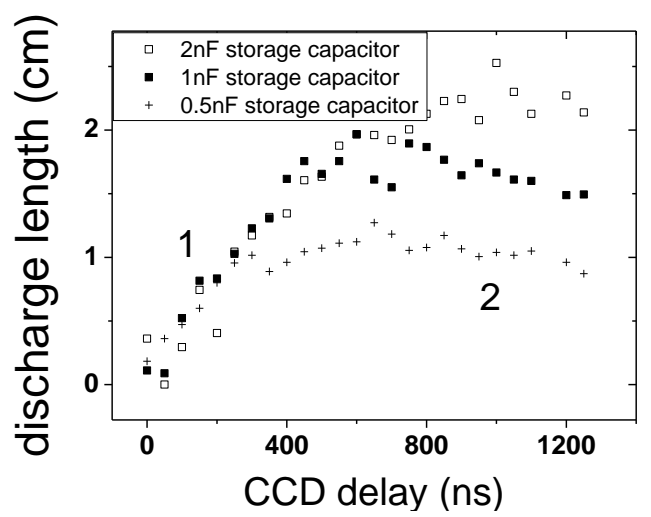

Figure 9a. Time dependence of the distance of the end of the filament from the pin electrode (radius defined on ), obtained with a single iCCD and accumulated images, the discharge size is plotted as a function of time for different applied voltage pulse duration, the propagation velocity is extracted by derivating phase (1), the discharge stopping length (2) depends on applied voltage decay, $(40 \mathrm{kV}, 4 \mathrm{~cm}$ gap, $100 \mu \mathrm{S} / \mathrm{cm})$.

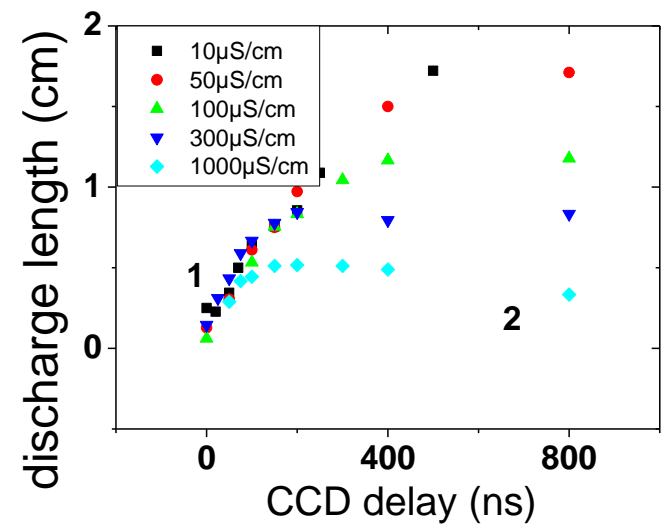

Figure 9b. Time dependence of the distance of the end of the filament from the pin electrode, influence of the liquid conductivity do not change the propagation velocity of the second positive mode, only the stopping length is modified because the applied voltage pulse duration is also modified when changing the water conductivity, see figure $14,(40 \mathrm{kV}, 4 \mathrm{~cm}$ gap).

\subsection{Discharge current, branching and morphology of the discharge}

The discharge morphology during the secondary mode is tree-like with several levels of branching. Typical branching patterns and its chaotic behaviour are presented in figure 5 and .

When branching occurs, an increase of the emission intensity has been observed in the parent filament in and figure 10. This emission intensity increase is likely to be related to an increase of the current flowing though the "parent filament" since it needs to supply more current for the "children filaments" at a branching node. The current of the primary mode propagation is in the $100 \mathrm{~mA}$ range and is lower than the current of the secondary mode propagation in the 1A range according to the velocity difference between those two modes as it would be the case in a purely displacement current. However in the case of water the current is always some order of magnitude higher compared to classical insulating liquids where the discharge propagates at similar velocities with an equivalent number of branches. Conductivity of oil is typically ten times lower than conductivity of distilled water which is typically $5 \mu \mathrm{S} / \mathrm{cm}$. Thus either the charge per filament is much higher in the case of water or there is a purely resistive behaviour.

An indication in favor of a resistive behaviour is given by testing the influence of the liquid conductivity on the discharge. At higher conductivity, the propagation velocity remains the same as mentioned previously figure $9 \mathrm{~b}$, and the number of filaments does not change much figure 11 , however the plasma current due to the propagation of the secondary mode is higher at higher conductivity . The emission intensity of the discharge is also increased at higher liquid conductivity figure 11. Thus the current per filament is higher at high water conductivity. 
Given this resistive behaviour, it is however surprising that the plasma propagation is not influenced by the liquid conductivity. If the deposition of energy is controlled by the gas side (plasma channel resistivity, charge accumulation at the interface) of the channel/liquid interface, an increased current should imply a higher propagation velocity which is not the case. And conversely, if the energy deposition is controlled by the liquid side (enhanced conductivity, space charge, double layer, or ballast resistor due to the water length between the filament tip and the opposite plane electrode), an increase in conductivity should have also some influence.

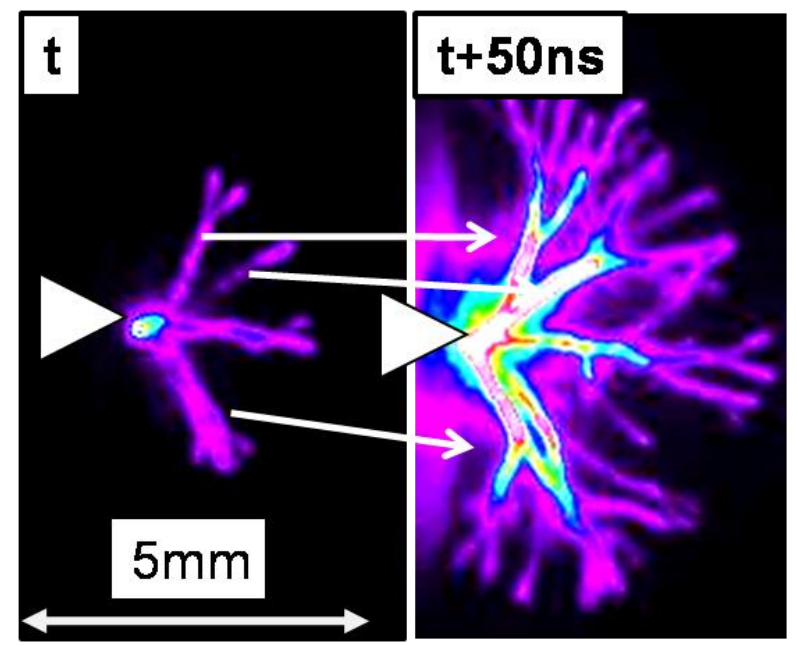

Figure 10. Emission intensity is linked to the number of children branches at a branching node. Branching leads to emission intensity jump. Pair of images taken on the same applied voltage pulse and obtained with the $2 \mathrm{iCCDs}$ setup with $2 \mathrm{~ns}$ gate and $50 \mathrm{~ns}$ difference, $(100 \mu \mathrm{S} / \mathrm{cm}, 40 \mathrm{kV}, 4 \mathrm{~cm}$ gap $)$.
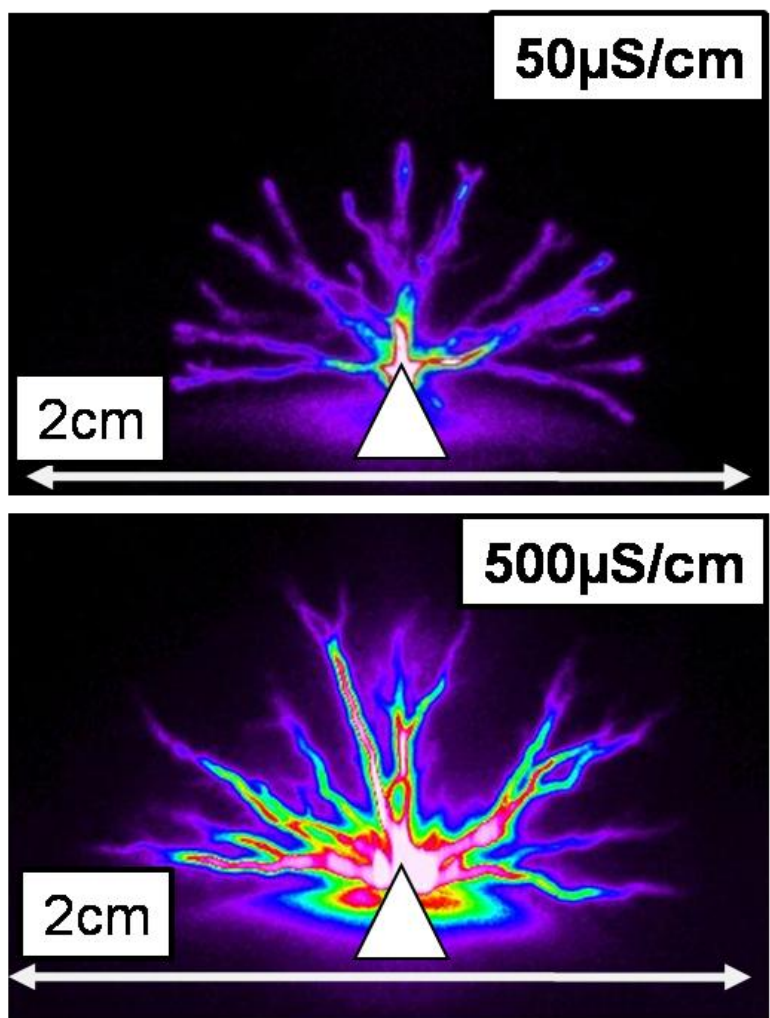

Figure 11. Influence to the liquid conductivity on the filament shape and the emission intensity. Emission intensity is much higher at large water conductivity. The filaments become triangular shaped, $(40 \mathrm{kV}, 4 \mathrm{~cm}$ gap). 


\subsection{Reilluminations: case of water at low conductivity}

At low water conductivity, after the successive propagation of the primary positive mode and the secondary positive mode, the discharge stops and restarts again as shown in figure 6, figure 12 and figure 13. The fact that the discharge is able to reilluminate and continues the propagation (even if the applied voltage has further decreased due to its RC decay shape) means that some charge deposition and electric field screening is going on at the plasma/liquid interface. This DBD-like mechanism (or Trichel-like) is suggested by the fact that the reillumination occurs only at low liquid conductivity figure $4 \mathrm{~b}$.

The current flowing through the reactor falls to the conduction level (1) after the stopping of the secondary mode propagation in figure $4 \mathrm{a}$ and figure $4 \mathrm{~b}$. It means that the filaments are no more conductive at that time. At higher water conductivity in figure $4 \mathrm{c}$, the removal of charges supposedly accumulated at the gas/liquid interface is faster than the deposition rate supplied by the plasma and no capacitive behaviour is possible. The plasma filaments stops to propagate at the stopping voltage as shown in figure 14 but some current continues to flow figure $4 \mathrm{c}$ and thus the filament continues to emit light according to the applied voltage decay during some microseconds. Reilluminations progressively disappear as the water conductivity is increased.

With emission imaging with an iCCD one can observe that the reillumination concerns only one filament at a time in figure 6 , not all the channels are reilluminated. The emission intensity of the reilluminating filament is weaker than the emission intensity of the secondary mode. However, the tip of the reilluminating filament is very luminous with emission intensity level identical to the emission intensity of the secondary mode. On nanosecond gate images this luminous tip can be just a spot or a segment as shown in figure 6 and figure 12. Each reillumination is associated with a current impulsion in (4) in figure $4 \mathrm{~b}$. The current impulsions are of several Amperes amplitude above the resistive conduction current due to water resistivity as shown in figure $4 \mathrm{~b}$. And they lasts a few tens of nanoseconds. The current spikes amplitude decreases over time during the applied voltage pulsed RC decay, and the repetition frequency does not seem to follow any pattern as shown in (4) in figure $4 \mathrm{~b}$. With the two iCCD setup one can observe that successive reilluminations concerns different channels. The reason why some particular channel reilluminates and not any other channel is not clear. This can be due to the different amount of charge deposited on this particular filament, or the different gas/liquid interface area of this specific filament.

The charge is likely to be deposited as adsorbed ions on the gas side of the gas/liquid interface or as solvated ion homocharge on the liquid side of the interface if the solvatation time scale of an adsorbed ion is lower that the removal time of solvated ion by conduction through the liquid. In the positive polarity, positive ions need to be adsorbed on the interface to explain the screening process. Those charges are deposited all along the filament and at the tip of the filament by drift-diffusion. The charge deposited along the filament side is likely to increase the plasma channel resistivity. The amount of charge required to screen the electric field will depends on the channel length, however we have seen that all the channels propagates at the same time thus the length difference from one channel to the next should be minimal. On the contrary, the removal rate can be faster on some channels due to a larger surface area of more branched channels. All the filaments turn off simultaneously at the end of the propagation of the secondary mode and turns on one at a time.

One should keep in mind that the gas channel produced by the plasma discharge propagation will start to expand, it means that the channel will cool down and the gas pressure will start to decrease when reillumination occurs. Thus the conduction of the channel can possibly be reestablished due to a channel diameter increase and not charge removal. The reillumination occurs in a gas channel according to the pressure*gap Paschen-like scaling law. Here the "gap" is defined between the pin electrode and the tip of the filament. Such pressure decrease can trigger the reillumination. Since the propagation velocity is supersonic, the pressure of the gas is not the same at the tip of the filament and closer to the pin electrode. The pressure decay is thus not the same for all filaments because it will depend on the energy release inside the filaments and the branching pattern. This would explain why reillumination of the several channels is not simultaneous.

With streak imaging one can observe that reillumination of the same filament happens several times as shown in figure 13. It means that the reillumination of one filament does not last long and is also stopped after some time. The reillumination duration lasts only a few tens of nanosecond as indicated by the current peaks in figure $4 \mathrm{~b}$. The fact that the propagation of the secondary mode lasts hundreds 
of nanoseconds instead of tens of nanoseconds for the reilluminations indicates that either this screening problem is more and more critical as filaments get longer or it happens when the applied voltage has decayed close to the end of the applied voltage pulse.

It is not clear on streak images in figure 13 if the reilluminating filament propagates further into the gap during the reillumination or from one reillumination to the next. With the 2 iCCDs experimental setup we were able to demonstrate that each reillumination is associated to the propagation of a new segment at the tip of the plasma filament, this can be qualified as a stepwise propagation in figure 12 . This step propagation (reillumination) happens after the main phase of the propagation (primary and then secondary mode) which is continuous in figure 8. During a same shot the discharge propagation thus shift from continuous to stepwise in figure 13 and this would explain the confusion of several studies reporting either continuous of stepwise propagation in water for similar experimental conditions.

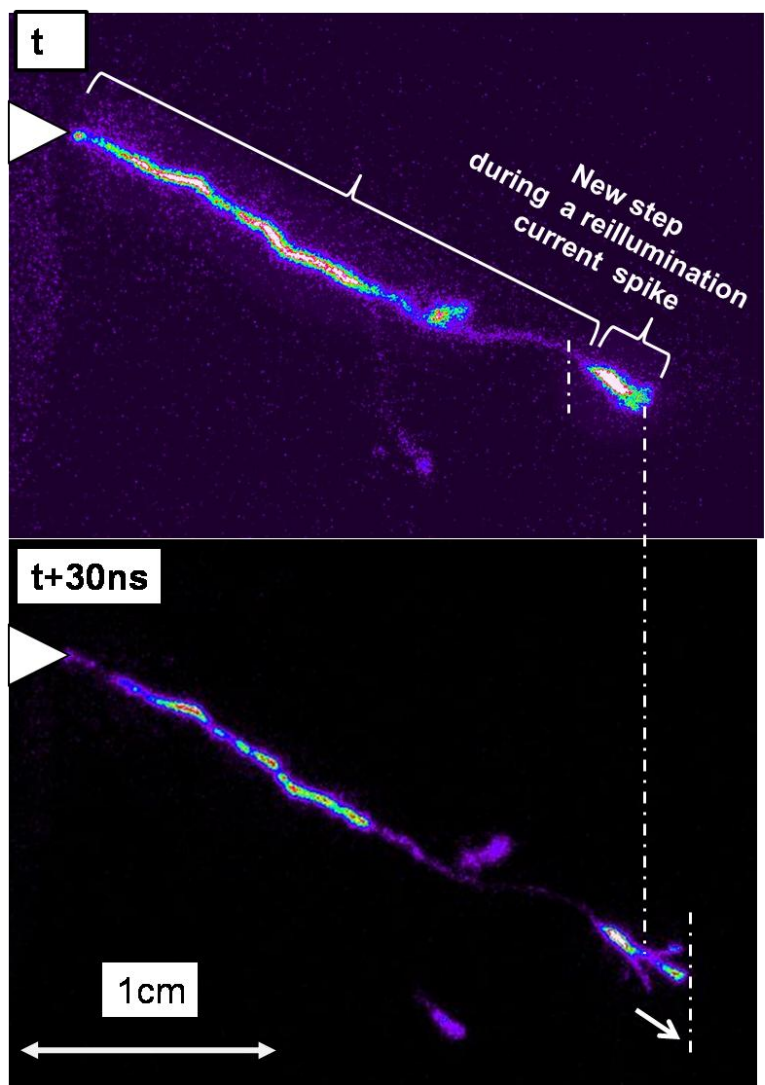

Figure 12. Reillumination in distilled water, pair of images taken on the same applied voltage pulse with the 2 iCCDs setup with the two cameras on each side of the reactor, the low intensity sections of the filament are thus not due to field of depth. Pair of images taken on the same applied voltage pulse with 2 ns gate and separated by $30 \mathrm{~ns}$ during a reillumination current spike, the reilluminating channel can be distinguished followed by a bright new section, a reillumination is thus a stepwise propagation. 


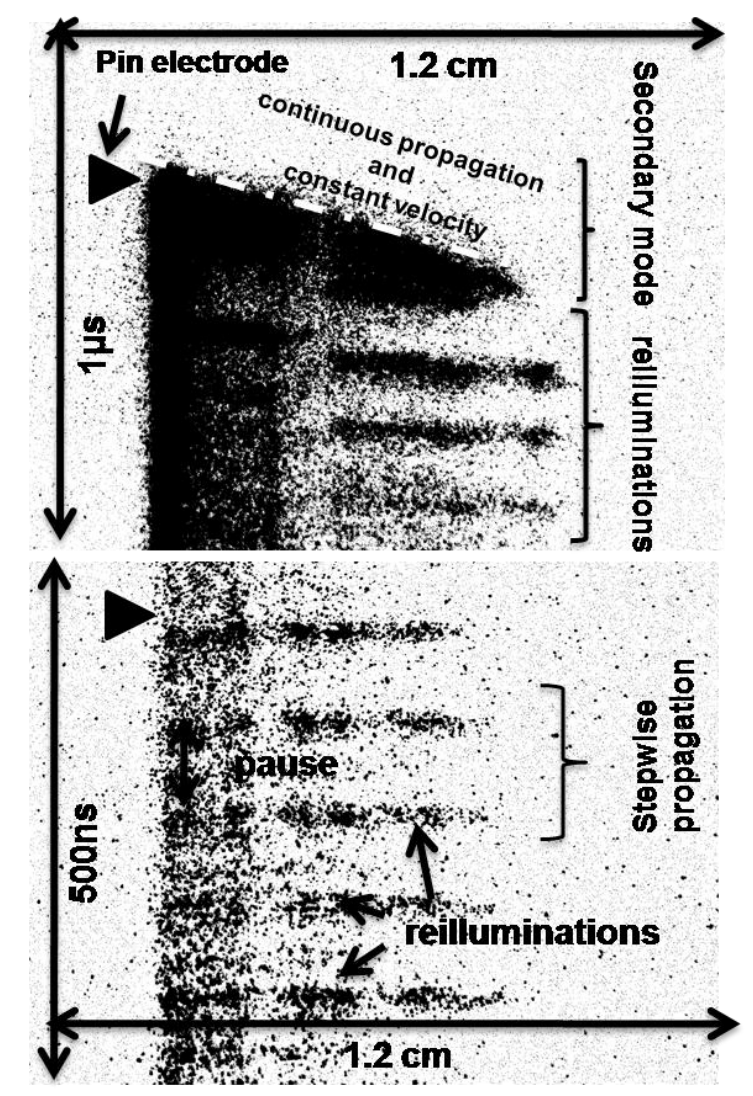

Figure 13. Streak image of the propagation of the secondary positive mode followed by reilluminations, (distilled water, $40 \mathrm{kV}, 4 \mathrm{~cm}$ gap).

\subsection{Stopping of the discharge}

The figure 9a also gives information about the "stopping length" of the discharge (2). The discharge stops to propagate at some length in the interelectrode gap, this stopping length is related to the applied voltage pulse duration. Increasing the pulse duration will not change the propagation velocity or the discharge shape: it will just change the propagation duration as can be seen by changing the value of the storage capacitor in the power supply in figure 9a. shows that the discharge propagates farther at low conductivity, however, the applied voltage pulse duration also changes because of lower reactor impedance when increasing the liquid conductivity. Similar pulse duration for different liquid conductivities can be obtained by changing the storage capacitor value to obtain the same pulse duration. One can observe that the stopping voltage is higher at lower liquid conductivity as shown in figure 14 .

This stopping voltage gives some criterion about the propagation sustainment in the liquid. It can mean that the plasma filament is more resistive at lower conductivity or that charges are more difficult to extract from a low conductivity liquid at the filament head. In either case one can wonder why the propagation velocity would stay the same whereas the stopping voltage would be different. A more resistive filament can be due to smaller filament diameter, or lower electronic density. It is difficult to distinguish any change in the filament radius based on emission imaging figure 11, shadow imaging would be more appropriate here. The emission intensity is however much brighter at higher liquid conductivity figure 11 which could indicate a higher electronic density. Spectroscopic measurements are required to verify this assumption on electronic density.

In fact the larger stopping voltage at low conductivity in figure 14 is to be related to the reilluminations that disappear almost completely above $200 \mu \mathrm{S} / \mathrm{cm}$. 


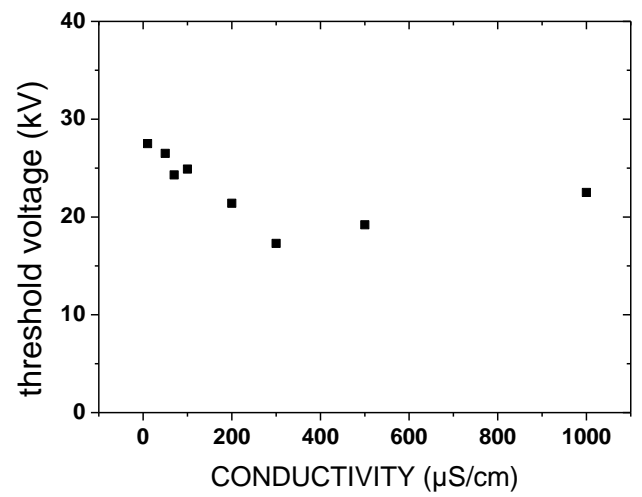

Figure 14. Stopping voltage as a function of the liquid conductivity, sustainment criterion for the discharge propagation, obtained from figure $9 \mathrm{~b}$.

\section{Discussion on the discharge propagation velocity behaviour as a function of applied voltage and as a function of the water conductivity}

Generally speaking, charged particle generation and motion is likely to be strongly influenced by the applied electric field according to the classical gas discharge theory. Basically, the voltage is required at the tip of the plasma filament to extract the electrons from the liquid (at positive applied voltage polarity), and to ensure the conduction of current trough the filaments in order to compensate for the losses and supply power to the propagating plasma filament tip.

The constant propagation velocity along the gap is counterintuitive since the Laplacian electric field (resulting from electrode geometry without plasma) strongly decreases along the interelectrode axis in pin to plane electrode geometry. However, this field decrease occurs on a scale equal to a few time the radius of curvature of the pin electrode and the electric field decreases more smoothly on the main part of the interelectrode gap. The plasma filament is also likely to be resistive and a significant voltage drop between the tip of the plasma filament and the HV electrode would appear as the filament grows longer. Thus if the propagation velocity of the discharge would be proportionally related to the voltage at the plasma filament tip one would observe a slowing down as the discharge propagates and gets longer.

The applied electric field is superimposed to the space charge electric field at plasma/liquid interface. The lack of influence of the applied voltage - once the initiation voltage is reached - can indicate that the propagation is ensured mainly by the space charge electric field at the tip of the plasma filament as in the case of gas-phase streamers. A sufficient voltage would then be required to launch the filaments but after that, the filament is self- sustained and any further increase of the voltage is not used for propagation but is likely used to form a larger number of plasma filaments that is to say branching. The larger charge is spited between more filaments. However, it is difficult to study branching in order to verify this last assumption. Branching pattern changes dramatically from one shot to another as shown in figure 5 and no clear law can be deduced from experiments. In particular, the individual segment length between two nodes or the number of individual filaments at each time has more variability between several shots in same conditions compared with changing the water conductivity or the applied voltage.

Given the timescale of the propagation, we cannot truly speak of a streamer-like mechanism. The gasphase streamer mechanism is an ionization wave which implies that ions remain static during the propagation. It is not the case here: the ions have time to move, taking ion mobility (over pressure) of $1 \mathrm{e} 6 \mathrm{~cm} 2 /$ torr $/ \mathrm{V} / \mathrm{s}$ with $1 \mathrm{MV} / \mathrm{cm}$ field and pressure in the range $10-1000$ bar we obtain $1 \mathrm{e} 5-1 \mathrm{e} 7 \mathrm{~cm} / \mathrm{s}$ which is very close to propagation velocity of $3 e 6 \mathrm{~cm} / \mathrm{s}$. A sheath has time to form and thus there will be with ion flux across the sheath potential. This ion flux can be of particular importance to explain the propagation. However it needs to be directed to explain the filamentary shape of the plasma filaments without lateral growth. The propagation can proceed by "etching" of the gas/liquid interface with secondary electron emission instead of electron extraction by field ionization as usually assumed in positive discharges in liquids. 
The fact that propagation velocity does not depends on the water conductivity is very surprising and counter intuitive since one could at first think that the propagation of a plasma discharge inside the liquid follows a vaporization process and that this vaporization should be favored by a more conductive liquid. The propagation velocity is linked to the energy deposition at the tip of the plasma filament. This energy deposition is thus not sensitive to the concentration of solvated ions and the higher current crossing the plasma/liquid interface. A local joule heating in front of the plasma filament tip occurs due to the conductive plasma filament, its radius of curvature and the solvated ions inside water. This heating is thus not responsible for the filament propagation or is regulated. The radius of curvature of the filament tip, namely its diameter is thus of particular importance here. The concentration of solvated ions (experimentally adjusted) inside water is perhaps not relevant because the plasma provide additional ions by charge injection or charge exchange at the plasma/liquid interface. A highly conductivity can be achieved in front of the plasma filament whatever the concentration of solvated ions from dissolved salt. This constant propagation velocity of the plasma discharge over two decades of liquid conductivity indicates that some self regulating or some limiting mechanism is going on.

The reported constant velocity across the gap and as a function of applied voltage and conductivity must be caused by a limiting or a self regulating mechanism: the space charge, the phase change or branching. Limitation can be caused by the electron extraction process from the liquid or the vaporization process. Electron extraction by field ionization entirely depends on the electric field and is thus not intrinsically limited. On the contrary, a secondary electron emission by ion impact on the water interface depends on the ion flux. This ion flux is driven by the power deposited in the plasma. The vaporization process is limited by the heating rate of the liquid and the nucleated cavity expansion. This cavity expansion depends on the driving pressure inside the nucleated cavity and on the liquid viscosity or volumic density. The heating rate depends on the energy feed from the pin electrode to the plasma filament tip and on the mechanism responsible for local energy deposition.

Auto-regulation can be explained by branching or by the filament resistivity. The branching can play an auto-regulation feedback by splitting the space charge into constant tip charges along the gap or by screening the electric field from one filament to another adjacent filament which would ensure a constant electric field at the plasma filament tips.

The plasma channel resistivity can also play a regulation role. The channel resistivity will increase as the filament propagates and the space charge amplification across the gap could be balanced. On one hand, any acceleration will be associated to a longer filament and thus a larger series resistance. On the other hand, the space charge will have difficulties to maintain its value because less power will be available through the longer resistive filament. Thus the space charge electric field will decrease and the propagation will slow down. The propagation velocity would be regulated by the power balance between the feed through a resistive filament and the power consumed for the propagation.

The discharge branching pattern or morphology would have a regulating influence on the propagation. At least, even if propagation velocity and the morphology (branching and number of filaments) of the discharge remain pretty much the same when increasing water conductivity, this increased power dissipation through the filament in the wake of the propagating tip should have some influence on the plasma parameters (ne, Te) and the gas pressure or temperature and thus modify strongly the induced chemistry [54].

\section{Conclusions}

- A precise time resolved imaging study of a plasma discharge in water was performed for positive applied voltage polarity. Emission of the plasma discharge has been observed with nanosecond imaging using two iCCDs and a streak camera. The detailed description of the different stages of the discharge growth has been described and analyzed as a function of an original experimental parameter: the water conductivity.

- The discharge begins with a non luminous initiation phase, followed by a primary, a secondary filamentary mode, and reilluminations before stopping or transition to spark.

- Initiation takes longer time than propagation an presents a huge jitter. Initiation time delay is consistent with nucleation of a microbubble at the initiation region by joule heating. 
- The propagation velocity of the secondary positive mode has been measured to be $30 \mathrm{~km} / \mathrm{s}$ and is continuous on nanosecond timescale resolution. This velocity remains constant during the propagation across the gap and surprisingly does not depend on the water conductivity.

- Reillumations of the gas channel have been observed at low water conductivity and present a DBD-like behaviour with a stepwise propagation.

\section{References}

[1] Gennady A. Mesyats, 2005, Pulsed Power, Springer

[2] Vasily Y. Ushakov et al, 2007, Impulse Breakdown of Liquids, Power Systems, Springer

[3] T.H. Martin, M. Williams, M. Kristiansen, 1996, Advances in Pulsed Power Technology

[4] Y.P.Raizer, 1991, Gas discharge Physics, Springer

[5] M. Pompili, C. Mazzetti, R. Bartnikas, 2005, Partial Discharge Pulse Sequence Patterns and Cavity Development Times in Transformer Oils under ac Conditions, IEEE Transactions on Dielectrics and Electrical Insulation 12 395-403

[6] H. M. Jones and E. E. Kunhardt, 1994, The Influence of Pressure and Conductivity on the Pulsed Breakdown of Water, IEEE Transactions on Dielectrics and Electrical Insulation 6 1016-25

[7] H M Jones and E E Kunhardt, 1995, Development of pulsed dielectric breakdown in liquids, $J$. Phys. D Appl. Phys. 28 178-88.

[8] J. Kolb, S. Kono, S. Xiao, B. Goan, XP. Lu, C. Bickes, M. Laroussi, R.P. Joshi, K.H. Schoenbach, 2003, water and propylene carbonate as storage and switching media in pulsed power systems, IEEE Transactions on Dielectrics and Electrical Insulation 715-18

[9] J F Kolb, R P Joshi, S Xiao and K H Schoenbach, 2008, Streamers in water and other dielectric liquids, J. Phys. D: Appl. Phys. 41

[10] B. R. Locke, M. Sato, P. Sunka, M. R. Hoffmann, J.-S. Chang, Electrohydraulic, 2006, Discharge and Nonthermal Plasma for Water Treatment, Ind. Eng. Chem. Res. 45 882-905

[11] Peter Bruggeman and Christophe Leys, 2009, TOPICAL REVIEW Non-thermal plasmas in and in contact with liquids, J. Phys. D: Appl. Phys. 42

[12] Petr Lukes, Martin Clupek, Vaclav Babicky, Vaclav Janda and Pavel Sunka, 2005, Generation of ozone by pulsed corona discharge over water surface in hybrid gas-liquid electrical discharge reactor,

J. Phys. D: Appl. Phys. 38 409-416

[13] Petr Lukes and Bruce R Locke, 2005, Plasmachemical oxidation processes in a hybrid gas-liquid electrical discharge reactor, J. Phys. D: Appl. Phys. 38 4074-81

[14] David R. Grymonpré, Amit K. Sharma, Wright C. Finney, Bruce R. Locke, 2001, The role of Fentons reaction in aqueous phase pulsed streamer corona reactors, Chemical Engineering Journal $\mathbf{8 2}$ 189-207

[15] Bing Sun, Masayuki Sato and J S Clements, 1999, Use of a pulsed high-voltage discharge for removal of organic compounds in aqueous solution, review, J. Phys. D: Appl. Phys. 32 1908-15 [16] B Sun, M sato, Clements, 2000, Oxidative Processes Occurring When Pulsed High Voltage Discharges Degrade Phenol in Aqueous Solution, Environ. Sci. Technol. 34 509-13

[17] Sunao Katsuki, Hidenori Akiyama, Amr Abou-Ghazala and Karl H. Schoenbach, 2002, Parallel Streamer Discharges Between Wire and Plane Electrodes in Water, IEEE Transactions on Dielectrics and Electrical Insulation 9 498-506

[18] Kenneth R Stalder, Donald F McMillen, JeanWoloszko, 2005, Electrosurgical plasmas, J. Phys. D: Appl. Phys. 38 1728-38

[19] W.G. Graham and K R Stalder, 2007, Plasmas in saline solution, Journal of Physics: Conference Series 71

[20] R. P. Joshi, J. Qian, G. Zhao, J. Kolb, and K. H. Schoenbach, E. Schamiloglu and J. Gaudet, 2004, Are microbubbles necessary for the breakdown of liquid water subjected to a submicrosecond pulse, Journal Of Applied Physics 96 5129-39

[21] Natalia Yu. Babaeva and Mark J. Kushner, 2008, Consequences of inhomogeneities on branching of streamers in high pressure gases, Escampig

[22] V. M. Atrazhev, E. G Dmitriev and I. T. lakubov, 1991, The Impact Ionization and Electrical Breakdown Strength for Atomic and Molecular Liquids, IEEE transactions on Electrical Insulation 26 586-91 
[23] V. M. Atrazhev I. V. Timoshkin, 1998, Transport of Electrons in Atomic Liquids in High Electric Fields, IEEE Transactions on Dielectrics and Electrical Insulation 5 450-7

[24] T. J. Lewis, 1994, Basic Electrical Processes in Dielectric Liquids, IEEE transactions on Dielectrics and Electrical Insulation 1 630-43

[25] T. J. Lewis, J. P. Llewellyn, M. J. van der Sluijs, J. Freestone and R. N. Hampton, 1996, A new model for electrical ageing and breakdown in dielectrics, International Conference on Dielectric Materials Measurements \&Applications Conference Publication 430 220-4

[26] T. J. Lewis, 1998, A New Model for the Primary Process of Electrical Breakdown in Liquids, IEEE Transactions on Dielectrics and Electrical Insulation 3 306-15

[27] T. J. Lewis, 2003, Breakdown Initiating Mechanisms at Electrode Interfaces in Liquids, IEEE Transactions on Dielectrics and Electrical Insulation 10 948-55

[28] P. E. Frayssines, O. Lesaint, N. Bonifaci, A. Denat, F. Devaux, 2003, Prebreakdown and Breakdown Phenomena Under Uniform Field in Liquid Nitrogen and Comparison with Mineral Oil, IEEE Transactions on Dielectrics and Electrical Insulation 10 970-6

[29] N. Bonifaci, A. Denat, V. M Atrazhev, 1995, Onset Voltage for Corona Pulses in Gaseous A under High Pressure and in Liquid, IEEE Transactions on Dielectrics and Electrical Insulation 2 13742

[30] N. Bonifaci, A. Denat and B. Malraison, 1997, Transport phenomena in point-plane geometry in dense argon, Journal of Electrostatics 40 91-6

[31] O. Lesaint, P. Gournay and R. Tobazcon, 1991, Investigations on Transient Currents Associated with Streamer Propagation in Dielectric Liquids, IEEE Transactions on Electrical Insulation 4 699707

[32] O. Lesaint and P. Gournay, 1994, Initiation and Propagation Thresholds of Positive Prebreakdown Phenomena in Hydrocarbon Liquids, IEEE Transactions on Dielectrics and Electrical Insulation 4 702-8

[33] O. Lesaint and G. Massala, 1998, Positive Streamer Propagation in Large Oil gaps Experimental Characterization of Propagation Modes, IEEE Transactions on Dielectrics and Electrical Insulation $5360-70$

[34] O. Lesaint and M Jung, 2000, On the relationship between streamer branching and propagation in liquids: influence of pyrene in cyclohexane, J. Phys. D: Appl. Phys. 33 1360-68

[35] G. Massala and O. Lesaint, 1998, Positive Streamer Propagation in Large Oil Gaps Electrical Properties of Streamers, IEEE Transactions on Dielectrics and Electrical Insulation 5 371-81 [36] W. G. Chadband, G. T. Wright, 1965, A pre-breakdown phenomenon in the liquid dielectric hexane, Brit. J. Appl. Phys.16 305

[37] W. G. Chadband and T. M. Sufian, 1985, experimental support for a model of positive streamer propagation in liquid insulation, IEEE Transactions on Electrical Insulation 20 239-46

[38] W. G. Chadband, 1988, The Ubiquitous Positive Streamer, IEEE Transactions on Electrical Insulation 23 697-706

[39] W G Chadband, 1991, Electrical breakdown-from liquid to amorphous solid, J. Phys. D. Appl. Phys. 24 56-64

[40] D. B. Watson, W. Heyes, K. C. Kao, J. H. Calderwood, 1965, Some Aspects of Dielectric Breakdown of Solids, IEEE Transactions on Electrical Insulation 1 30-7

[41] P. K. Watson, 1985, Bubble growth following a localized electrical discharge and its relationship to the breakdown of triggered spark gaps in liquids, IEEE Transactions on Electrical Insulation 20 275-80

[42] P. Keith Watson and W. G. Chadband, 1988, The Dynamics of Pre-Breakdown Cavities in Viscous Silicone Fluids in Negative Point-Plane Gaps, IEEE Transactions on Electrical Insulation 23 729-38

[43] P. Keith Watson, W. G. Chadband, M. Sadeghzadeh-Araghi, 1991, The Role of Electrostatic and Hydrodynamic Forces in the Negative-point Breakdown of Liquid Dielectrics, IEEE Transactions on Electrical Insulation 26 543-59

[44] I. Foffana, A. Beroual, 1998, predischarge models in dielectric liquids, Jpn J Appl Phys 37 254047

[45] A. Beroual, C. Marteau and R. Tobazcon, 1988, Behaviour of Streamers in Liquids under Step Voltages in Point-Plane Geometry, IEEE Transactions on Electrical Insulation 23 955-959 
[46] Beroual, C. Marteau and R. Tobazeon, 1988, Measurement of the Charge of a Single or of a Series of Streamers in Liquids Subjected to a Voltage Step, IEEE transactions on Electrical Insulation 23 427-31

[47] A. Beroual, 1999, spectral analysis of light emitted by streamers and gas chromatography in liquid dielectric, Jpn J Appl Phys 32 5615-20

[48] A. Beroual, 1998, Propagation and Structure of Streamers in liquid Dielectrics, IEEE Electrical Insulation Magazine 14 6-17

[49] A. Beroual, 2002, influence of additives and hydrostatic pressure on streamers initiation and dielectric strength of liquids, annual report conference on electrical insulation and dielectric phenomena $248-51$

[50] S Ingebrigtsen, L E Lundgaard and P-O Åstrand, 2007, Effects of additives on prebreakdown phenomena in liquid cyclohexane: II. Streamer propagation, J. Phys. D: Appl. Phys. 41

[51] A A Kulikovsky, 1997, Positive streamer between parallel plate electrodes in atmospheric pressure air, J. Phys. D: Appl. Phys. 30 441-50

[52] A. A. Kulikovsky, 1998, Positive streamer in a weak field in air: A moving avalanche-to-streamer transition, Physical Review E. 57 7066-74

[53] N. J. Felici, 1988, Blazing a Fiery Trail with the Hounds, IEEE Transactions on Electrical Insulation 23 497-503

[54] P Sunka, V Babicky, M C lupek, P Lukes, M Simek, J Schmidt and M Cernak, 1999, Generation of chemically active species by electrical discharges in water, Plasma Sources Sci. Technol. 8 258-65 [55] Wladimir An, Kurt Baumung, and Hansjoachim Bluhm, 2007, Underwater streamer propagation analyzed from detailed measurements of pressure release, Journal Of Applied Physics 101 